\title{
Stabilization techniques and a posteriori error estimates for the obstacle problem
}

\author{
Dirk Biermann - Heribert Blum • Ivan Iovkov • Nicole Klein · Andreas \\ Rademacher • Franz-Theo Suttmeier
}

Received: date / Accepted: date

\begin{abstract}
This work deals with a posteriori error estimates for the obstacle problem. Deriving an estimator on the basis of the variational inequality with respect to the primal variable, an inconsistent one is obtained. To achieve consistency, this problem is treated by a Lagrange formalism, which transfers the variational inequality into a saddle point problem. Different techniques to ensure the stability of the discretization and to solve the discrete problems by iterative solvers are studied and compared. Numerical tests confirm our results of consistent a posteriori error estimation.
\end{abstract}

Keywords obstacle problem - finite element method . stabilization $\cdot$ a posteriori error estimate $\cdot$ variational inequality

\section{Introduction}

In the mathematical modelling of many engineering applications, contact problems play an important role. Let us shortly consider a drilling process as an example:

\section{Biermann · I. Iovkov}

Institute of Machining Technology, Technische Universität Dortmund, Baroper Straße 301, 44227 Dortmund, Germany

E-mail: biermann@isf.de, iovkov@isf.de

H. Blum · A. Rademacher

Institute of Applied Mathematics, Technische Universität Dortmund, Vogelpothsweg 87, 44227 Dortmund, Germany

E-mail: heribert.blum@tu-dortmund.de,

andreas.rademacher@tu-dortmund.de

N. Klein · F.-T. Suttmeier

Technische und Naturwissenschaftliche Fakultät, Universität Siegen, Walter-Flex-Straße 3, 57068 Siegen, Germany

Tel.: +49-271-740-3516

Fax: +49-271-740-3632

E-mail: nicole.klein.83@gmail.com,

info@fem2m.de
There, the decisive forces and heat flows are generated by the frictional contact between the drill and the workpiece. An accurate mathematical modelling of the contact situation and a precise numerical simulation of it are indispensable to obtain a reasonable simulation of the process. The contact zone between the drill and the workpiece is a $2 \mathrm{D}$ surface. An adequate model problem to study the behaviour of the contact surface is given by $2 \mathrm{D}$ obstacle problems. In this article, we develop numerical algorithms by means of this model problem.

Taking a domain $\Omega \subset \mathbb{R}^{2}$, the strong formulation of the obstacle problem reads

$$
\begin{gathered}
-\Delta u-f \geq 0, \\
u-\psi \geq 0, \\
(u-\psi)(-\Delta u-f)=0
\end{gathered}
$$

in $\Omega$ with $u: \Omega \rightarrow \mathbb{R}$ sufficiently smooth and the Dirichlet boundary condition $u=0$ on $\partial \Omega$. Here, $f: \Omega \rightarrow \mathbb{R}$ represents the prescribed body forces and $\psi: \Omega \rightarrow \mathbb{R}$ the obstacle. The Laplace operator $\Delta$ is defined by $\Delta u=\frac{\partial^{2} u}{\partial x^{2}}+\frac{\partial^{2} u}{\partial y^{2}}$

To use finite element techniques we rewrite the system (1-3) as a variational inequality using integration by parts: Find $u \in K$ with

$(\nabla u, \nabla(\varphi-u)) \geq(f, \varphi-u) \quad \forall \varphi \in K$,

where we set $V:=H_{0}^{1}(\Omega)$ and $K=\{v \in V \mid v \geq$ $\psi$ a.e. in $\Omega\}$ and assume $\psi \in H^{1}(\Omega)$ as well as $f \in$ $L^{2}(\Omega)$. Here and in what follows, $(\cdot, \cdot)$ represents the $L^{2}:=L^{2}(\Omega)$ inner product and $\|\cdot\|$ the corresponding norm. Furthermore $H^{m}:=H^{m}(\Omega)$ denotes the standard Sobolev space of $L^{2}$-functions with weak derivatives in $L^{2}$ up to the order $m$, and $H_{0}^{1} \subset H^{1}$ is the subspace of $H^{1}$-functions with vanishing trace on $\partial \Omega$. 
The variational inequality (4) is uniquely solvable, cf. [17].

Next, we present the Lagrange fomulation of problem (4): Find a pair $(u, \lambda) \in V \times \Lambda$ with

$\mathcal{L}(u, \lambda)=\inf _{\varphi \in V} \sup _{w \in \Lambda} \mathcal{L}(\varphi, w)$,

where $\Lambda:=\left\{q \in L^{2} \mid q \geq 0\right.$ a.e. $\}$ and

$\mathcal{L}(\varphi, w):=\frac{1}{2}(\nabla \varphi, \nabla \varphi)-(f, \varphi)-(w, \varphi-\psi)$

The stationarity condition for a saddle point leads to the mixed problem: Find a pair $(u, \lambda) \in V \times \Lambda$ fulfilling the mixed formulation

$$
\begin{aligned}
(\nabla u, \nabla \varphi)-(\lambda, \varphi) & =(f, \varphi) \quad \forall \varphi \in V \\
(u, w-\lambda) & \geq(\psi, w-\lambda) \quad \forall w \in \Lambda .
\end{aligned}
$$

The existence of a unique solution $(u, \lambda)$ of $(7-8)$ and the equivalence of (4) with (7-8) are proven in [11].

The scope of the work at hand is to study different discretization methods for the mixed problem (7$8)$. There, it is crucial to ensure the stability and the uniqueness for the primal as well as for the dual variable of the discrete solution. We present two different approaches to guarantee stability. The first one is to balance the discrete counterparts of $V$ and $\Lambda$. In the second approach, we add a consistent stabilization term to inequality (8) and use the same discrete trial space $V_{h}$ for approximating $V$ and $\Lambda$. Furthermore, we discuss several iterative solution techniques for the discrete problems. The discretization schemes are presented in Section 2 and the numerical results in the Sections 4.1 and 4.2. For an overview of discretization schemes and solution algorithms of the discrete problems, we refer to $[16,24,25]$.

The second part of this article is devoted to a posteriori error estimation. Here, we are interested in the derivation of consistent a posteriori error estimates for the obstacle problem. For comparison reasons, we show an inconsistent error estimator on the basis of the variational inequality (4) in Theorem 1 . To obtain a consistent estimator, the mixed formulation (7-8) is chosen as starting point. The corresponding result is presented in Theorem 2. When we include the consistent stabilization term in the problem formulation, the a posteriori error estimate has to be slightly modified, see Theorem 3. Finally, in Theorem 4 we discuss the a posteriori error estimates for the obstacle problem given in [19] or [4], respectively, where an auxiliary problem taken from (78 ) is the basis for the estimate. Comparative numerical results w.r.t. the a posteriori error estimates are presented in Section 4.3. Besides the techniques presented in this paper to derive a posteriori error estimates, a lot of contributions, which use different techniques, exist, see e.g. $[1,2,9,12,15,18,22,23]$. The convergence of adaptive finite element schemes for obstacle problems is studied in $[5,6,21]$.

\section{Discretisation}

We apply the finite element method to compute an approximate solution of (4) on decompositions $\mathbb{T}_{h}=$ $\left\{T_{i} \mid 1 \leq i \leq N_{h}\right\}$ of $\Omega$ consisting of $N_{h}$ quadrangular elements $T_{i}$, satisfying the usual conditions of shape regularity. The width of the mesh $\mathbb{T}_{h}$ is characterised in terms of a piecewise constant mesh size function $h=h(x)>0$, where $h_{T}:=h_{\mid T}=\operatorname{diam}(T)$. Thus the approximate solution $u_{h}$ of the discrete inequality is characterised by

$\left(\nabla u_{h}, \nabla\left(\varphi-u_{h}\right)\right) \geq\left(f, \varphi-u_{h}\right) \quad \forall \varphi \in K_{h}$,

where $K_{h}$ is a closed and convex subset of the standard finite element space $V_{h}$ based on $\mathbb{T}_{h}$ and bilinear shape functions. Furthermore, $K_{h}$ is chosen as an appropriate discrete substitute of $K$, i.e. $K_{h}=\left\{v \in V_{h} \mid v \geq \psi_{h} \in\right.$ $\Omega$, where $\psi_{h} \in V_{h}$ is the bilinear interpolant of $\psi$. For the sake of simplicity, we assume $\psi=\psi_{h}$. We solve the arising discrete system by the cG-PSSOR method, cf. [3].

We are especially interested in a posteriori error estimation. For a priori error bounds see, for instance, [8] or $[10]$.

The discrete version of the saddle point problem (7)(8) reads

$$
\begin{aligned}
\left(\nabla u_{h}, \nabla \varphi\right)-\left(\lambda_{H}, \varphi\right) & =(f, \varphi) \quad \forall \varphi \in V_{h} \\
\left(u_{h}, w-\lambda_{H}\right) & \geq\left(\psi, w-\lambda_{H}\right) \quad \forall w \in \Lambda_{H} .
\end{aligned}
$$

Here, $\Lambda_{H}:=L_{H} \cap \Lambda$ and $L_{H}$ is a finite element space on a possibly different mesh $\mathbb{T}_{H}$. We will precisely specify $L_{H}$ after the presentation of the solution algorithms.

The system (10)-(11) in matrix vector notation reads

$$
A \bar{u}+B \bar{\lambda}=F
$$

$\forall \bar{\mu} \in \bar{\Lambda}: \quad(\bar{\mu}-\bar{\lambda})^{\top}\left(B^{\top} \bar{u}-\bar{\psi}\right) \leq 0$

with the usual stiffness matrix $A$ and right hand side $F$. The pairing of $\lambda$ and $u$ is represented by $B$. Furthermore, we choose $\bar{\Lambda}:=\mathbb{R}_{+}^{n}$. One way to solve the system (12)-(13) is the use of a Uzawa-type-algorithm:

Choose an initial iterate $\bar{\lambda}^{0}$ and $\alpha>0$. For $s=1,2, \ldots$ :

$$
\begin{aligned}
A \bar{u}^{s} & =f-B^{\top} \bar{\lambda}^{s-1}, \\
\bar{\lambda}^{s} & =\max \left(0, \bar{\lambda}^{s-1}+\alpha\left(B \bar{u}^{s}-\bar{\psi}\right)\right)
\end{aligned}
$$


An alternative is given by using the Schur complement.

This leads to the quadratic minimisation problem

$Z(\bar{\lambda})=\min _{\bar{\mu} \in \bar{\Lambda}} Z(\bar{\mu})$

$$
A \bar{u}+B \bar{\lambda}=F
$$

$\forall \bar{\mu} \in \bar{\Lambda}:$

with

$Z(\bar{\mu}):=\frac{1}{2} \bar{\mu}^{\top} B^{\top} A^{-1} B \bar{\mu}-\bar{\mu}^{\top}\left(B^{\top} A^{-1} F-\bar{\psi}\right)$

and $\bar{u}=A^{-1}(F-B \bar{\lambda})$. The matrix $B^{\top} A^{-1} B$ is positive semidefinit and we apply an active set method to solve the optimisation problem.

To obtain a stable solution $\left(u_{h}, \lambda_{H}\right)$, we have to ensure the inf-sup condition. From the experiences with Signorini problems, cf. [20], we know that using bilinear elements on the same mesh to discretise $\lambda$ does not lead to a stable discretisation. However, applying piecewise constant shape functions together with $H=2 h$ result in a stable discretisation, see Section 4.1.

Another possible way to obtain stability is to use the Galerkin least-square method by adding a consistent stabilization term like proposed by Hughes, et al. [13] and theoretically analysed by Franca and Stenberg [14]. Therefore, we choose the same triangulation and shape functions for $V_{h}$ and $L_{H}, h=H$. We know $\Delta u+\lambda+f$ to be equal to zero. The continuous system then reads

$$
\begin{aligned}
(\nabla u, \nabla \varphi)-(\lambda, \varphi) & =(f, \varphi) \\
(u, w-\lambda)+(\Delta u+\lambda, \delta(w+\Delta \varphi) & \geq \\
(\psi, w-\lambda) & -(f, \delta(w+\Delta \varphi))
\end{aligned}
$$

for all $\varphi \in V$ and all $w \in \Lambda$, where $\delta$ is a piecewise constant postitive parameter function. In the discrete system $u$ is interpolated by bilinear functions and since we have regular quadraliterals the terms $\Delta u_{h}$ and $\Delta \varphi$ disappear. If the grid is warped neglecting $\Delta u_{h}$ and $\Delta \varphi$ cause an interpolation error of the order $\mathcal{O}(\delta)$. Numerical tests show that $\delta$ ought to be chosen by $\delta=\gamma h^{2}$ with a positive constant $\gamma$ to garantee optimal stability. For the factor $h$ it is expedient to take the diameter of the active cell. So the interpolation error can be neglected because our error estimator is of the order $\mathcal{O}\left(h^{\frac{1}{2}}\right)$ as we will see in section 3 . We obtain

$$
\begin{aligned}
\left(\nabla u_{h}, \nabla \varphi\right)-\left(\lambda_{h}, \varphi\right) & =(f, \varphi) \quad \forall \varphi \in V_{h} \\
\left(u_{h}, w-\lambda_{h}\right)-\delta\left(\lambda_{h}, w\right) & \geq \\
\left(\psi, w-\lambda_{h}\right) & +\delta(f, w) \quad \forall w \in \Lambda_{h} .
\end{aligned}
$$

Solving this problem we get another mass-matrix $C$. The matrix vector notation (12), (13) now looks as follows:

Choosing the Uzawa-algorithm described in (14) we get the extended version:

$$
\begin{aligned}
A \bar{u}^{s} & =f-B^{\top} \bar{\lambda}^{s-1}, \\
\bar{\lambda}^{s} & =\max \left(0, \bar{\lambda}^{s-1}+\alpha\left(B \bar{u}^{s}-\bar{\psi}-\delta\left(C \bar{\lambda}^{s-1}+f\right)\right)\right) .
\end{aligned}
$$

In dependence of the chosen stabilization technique, there are several advantages and disadvantages. Choosing the second one by adding a stabilization term, the problem arises to determine the constant factor $\delta$ typically experimentally. Furthermore we get a modified matrix-vector multiplication in our solver, which runs very robust, even on irregular meshes. The shape functions of the Lagrange multiplier can be chosen constant or even bilinear.

Stabilizing the discretization by using piecewise constant trial functions on patches for the Lagrange multiplier involve some problems: The calculation has to be carried out on different meshes, especially a patch mesh must be generated, which may be difficult when using a mesh generator. Furthermore, the resolution of the contact situation is connected to the patch mesh and not as accurate as it could be in view of the mesh for the displacement. However, the creation of the patch is easy, if hierarchic meshes are used. This is mostly the case in adaptive finite elements. The stabilization approach can also be used for higher order finite elements, see $[19,20]$.

\section{A posteriori error analysis}

In this section, we present four different residual type a posteriori error estimates for the obstacle problem. The first one is derived on the basis of the variational inequality and is inconsistent. To deduce consistent a posteriori estimates, we use the mixed formulation of the obstacle problem as starting point. It turns out that the consideration of the Lagrange multiplier in the residual is crucial to obtain consistency.

In the following, we frequently need the standard Clément interpolation operator $i_{h}: H^{1}(\Omega) \rightarrow V_{h}$. For a function $v \in H^{1}(\Omega)$, the application of $i_{h}$ is shortly denoted by $v_{i}:=i_{h} v$. Let $\|v\|_{B}:=\left(\int_{B} v^{2} d x\right)^{1 / 2}$ be the $L^{2}$-Norm of $v$ on $B, B \subset \Omega$. We define $\omega_{x_{j}}:=$ $\cup\left\{T^{\prime} \in \mathbb{T}_{h} \mid x_{j} \in T^{\prime}\right\}$ if $x_{j}$ is a vertex of a mesh cell 
and $\tilde{\omega}_{T}:=\cup\left\{\omega_{x_{j}} \mid x_{j} \in T\right\}$. For the interpolation error, the estimates

$$
\begin{aligned}
\left\|v-v_{i}\right\|_{T} & \leq C_{i, T} h_{T}\|\nabla v\|_{\tilde{\omega}_{T}} \\
\left\|v-v_{i}\right\|_{\partial T} & \leq C_{i, \partial T} \sqrt{h_{\partial T}}\|\nabla v\|_{\tilde{\omega}_{T}}
\end{aligned}
$$

hold for all mesh cells $T \in \mathbb{T}_{h}$.

The error function is denoted by $e:=u-u_{h}$. We are interested in the estimation of the error in the energy norm of the obstacle problem, which is given by the $H^{1}$ seminorm $|e|_{1}:=(\nabla e, \nabla e)^{1 / 2}$. We start the derivation of the a posteriori error estimate by estimating

$$
\begin{aligned}
\left(\nabla e, \nabla e_{i}\right)= & \underbrace{\left(f, e_{i}\right)-\left(\nabla u_{h}, \nabla e_{i}\right)}_{\leq 0} \\
& +\left(\nabla u, \nabla\left(e_{i}-e\right)\right)-\left(f, e_{i}-e\right) \\
& +\underbrace{(\nabla u, \nabla e)-(f, e)}_{\leq 0} \\
\leq & \left(\nabla u, \nabla\left(e_{i}-e\right)\right)-\left(f, e_{i}-e\right) .
\end{aligned}
$$

Here, $(\nabla u, \nabla e)-(f, e) \leq 0$ is obtained, since we can test (4) by $\varphi=u_{h}$. Furthermore, $u_{i} \in K_{h}$ is an admissible test function in (9) and consequently $\left(f, e_{i}\right)-$ $\left(\nabla u_{h}, \nabla e_{i}\right)=\left(f, u_{i}-u_{h}\right)-\left(\nabla u_{h}, \nabla\left(u_{i}-u_{h}\right)\right) \leq 0$. Now we can easily estimate the square of the energy error

$$
\begin{aligned}
(\nabla e, \nabla e)= & \left(\nabla e, \nabla\left(e-e_{i}\right)\right)+\left(\nabla e, \nabla e_{i}\right) \\
\leq & \left(\nabla u, \nabla\left(e-e_{i}\right)\right)-\left(\nabla u_{h}, \nabla\left(e-e_{i}\right)\right) \\
& +\left(\nabla u, \nabla\left(e_{i}-e\right)\right)-\left(f, e_{i}-e\right) \\
= & \left(f, e-e_{i}\right)-\left(\nabla u_{h}, \nabla\left(e-e_{i}\right)\right) .
\end{aligned}
$$

Cell-wise integration by parts results in

$$
(\nabla e, \nabla e) \leq \sum_{T \in \mathbb{T}_{h}} \omega_{T} \rho_{T}
$$

with local residuals $\rho_{T}$ and weights $\omega_{T}$ defined by

$$
\begin{aligned}
\rho_{T} & :=h_{T}\left\|f+\Delta u_{h}\right\|_{T}+\frac{1}{2} h_{\partial T}^{\frac{1}{2}}\left\|n \cdot\left[\nabla u_{h}\right]\right\|_{\partial T}, \\
\omega_{T} & :=\max \left\{h_{T}^{-1}\left\|e-e_{i}\right\|_{T}, h_{\partial T}^{-\frac{1}{2}}\left\|e-e_{i}\right\|_{\partial T}\right\},
\end{aligned}
$$

where $\left[\partial_{n} u_{h}\right]$ denotes the jump of the normal derivative $\partial_{n} u_{h}$ for interior interelement boundaries and is set to zero on $\partial \Omega$ due to the homogeneous Dirichlet boundary conditions.

The interpolation error estimates (22) and (23) yield the following estimate for the discretisation error in the energy norm:

Theorem 1 There holds the a posteriori error bound

$|e|_{1}^{2} \leq C \eta\left(u_{h}\right):=C \sum_{T \in \mathbb{T}_{h}} \rho_{T}^{2}$

with local residuals $\rho_{T}$ defined in (24).
The a posteriori error estimate (26) can be improved by applying an interpolation error estimate, which takes the effect into account that the interpolation error is zero in the interior of the active contact set, if $\psi=\psi_{h}$.

Now, we give a precise definition of consistency for a posteriori error estimators:

Definition 1 An error estimator $\eta\left(u_{h}\right)$ is called consistent, if $\eta(u)=0$, i.e. if $\eta$ is evaluated for the continuous solution $u$ of the problem, then $\eta$ vanishes.

So looking at the definition of $\eta$ in Theorem 1, we notice the error estimator not to be consistent. In regions of contact, there may hold $\Delta u+f>0$, which determines the contact forces.

To obtain a consistent a posteriori error estimator, we make use of the Lagrange formalism and start again with estimating $\left(\nabla e, \nabla e_{i}\right)$ by

$$
\begin{aligned}
\left(\nabla e, \nabla e_{i}\right)= & \left(f, e_{i}\right)-\left(\nabla u_{h}, \nabla e_{i}\right)+\left(\nabla u, \nabla\left(e_{i}-e\right)\right) \\
& -\left(f, e_{i}-e\right)+(\nabla u, \nabla e)-(f, e) \\
\leq & -\left(\lambda_{H}, e_{i}\right)+\left(\nabla u, \nabla\left(e_{i}-e\right)\right)-\left(f, e_{i}-e\right) .
\end{aligned}
$$

Here, we make use of the fact that we can test (4) by $\varphi=u_{h}$ to obtain $(\nabla u, \nabla e)-(f, e) \leq 0$ as well as of (10). Taking this result we go on estimating:

$$
\begin{aligned}
\left(\nabla e, \nabla e_{i}\right) \leq & \left(\nabla u, \nabla\left(e_{i}-e\right)\right)-\left(f, e_{i}-e\right) \\
& -\left(\lambda_{H}, e_{i}+e-e\right) \\
= & \left(\nabla u, \nabla\left(e_{i}-e\right)\right)-\left(f, e_{i}-e\right) \\
& +\left(\lambda_{H}, e-e_{i}\right)-\left(\lambda_{H}, e\right) \\
= & \left(\nabla u, \nabla\left(e_{i}-e\right)\right)-\left(f, e_{i}-e\right) \\
& +\left(\lambda_{H}, e-e_{i}\right) \\
& -\left(\lambda_{H}, u-u_{h}-\psi+\psi\right) \\
= & \left(\nabla u, \nabla\left(e_{i}-e\right)\right)-\left(f, e_{i}-e\right) \\
& +\left(\lambda_{H}, e-e_{i}\right)+\left(\lambda_{H}, \psi-u\right) \\
& +\left(\lambda_{H}, u_{h}-\psi\right) \\
\leq & \left(\nabla u, \nabla\left(e_{i}-e\right)\right)-\left(f, e_{i}-e\right) \\
& +\left(\lambda_{H}, e-e_{i}\right)+\left(\lambda_{H}, u_{h}-\psi\right) .
\end{aligned}
$$

Now we can estimate the error in the energy-norm:

$$
\begin{aligned}
(\nabla e, \nabla e)= & \left(\nabla e, \nabla\left(e-e_{i}\right)\right)+\left(\nabla e, \nabla e_{i}\right) \\
\leq & \left(\nabla u, \nabla\left(e-e_{i}\right)\right)-\left(\nabla u_{h}, \nabla\left(e-e_{i}\right)\right) \\
& +\left(\nabla u, \nabla\left(e_{i}-e\right)\right)-\left(f, e_{i}-e\right) \\
& -\left(\lambda_{H}, e-e_{i}\right)+\left(\lambda_{H}, u_{h}-\psi\right) \\
= & \left(f, e-e_{i}\right)-\left(\nabla u_{h}, \nabla\left(e-e_{i}\right)\right) \\
& +\left(\lambda_{H}, e-e_{i}\right)+\left(\lambda_{H}, u_{h}-\psi\right) .
\end{aligned}
$$

Cell-wise integration by parts results in

$$
(\nabla e, \nabla e) \leq \sum_{T \in \mathbb{T}} \omega_{T} \varrho_{T}+N
$$


with local residuals $\varrho_{T}$ and weights $\omega_{T}$ defined by

$$
\begin{aligned}
\varrho_{T}:= & h_{T}\left\|f+\lambda_{H}+\Delta u_{h}\right\|_{T} \\
& +\frac{1}{2} h_{\partial T}^{\frac{1}{2}}\left\|n \cdot\left[\nabla u_{h}\right]\right\|_{\partial T}, \\
\omega_{T}:= & \max \left\{h_{T}^{-1}\left\|e-e_{i}\right\|_{T}, h_{\partial T}^{-\frac{1}{2}}\left\|e-e_{i}\right\|_{\partial T}\right\}, \\
N:= & \left(\lambda_{H}, u_{h}-\psi\right) .
\end{aligned}
$$

Next one uses the interpolation estimates (22) and (23) to get the following

Theorem 2 For the mixed FE-scheme (10)-(11) there holds the a posteriori error bound

$|e|_{1}^{2} \leq C \sum_{T \in \mathbb{T}_{h}} \varrho_{T}^{2}+N$

with $\varrho_{T}$ from (29) and $N$ from (31).

Let us consider the stabilized approximation scheme given in (18) and (19). The error estimator (32) only changes in the term $N$. Looking at (27) we now receive:

$$
\begin{aligned}
\left(\nabla e, \nabla e_{i}\right) \leq & \left(\nabla u, \nabla\left(e_{i}-e\right)\right)-\left(f, e_{i}-e\right) \\
& -\left(\lambda_{h}, e_{i}+e-e\right) \\
= & \left(\nabla u, \nabla\left(e_{i}-e\right)\right)-\left(f, e_{i}-e\right) \\
& +\left(\lambda_{h}, e-e_{i}\right)-\left(\lambda_{h}, e\right) \\
= & \left(\nabla u, \nabla\left(e_{i}-e\right)\right)-\left(f, e_{i}-e\right) \\
& +\left(\lambda_{h}, e-e_{i}\right)-\left(\lambda_{h}, u-u_{h}-\psi+\psi\right. \\
& \left.+\delta\left(f+\lambda_{h}\right)-\delta\left(f+\lambda_{h}\right)\right) \\
= & \left(\nabla u, \nabla\left(e_{i}-e\right)\right)-\left(f, e_{i}-e\right) \\
& +\left(\lambda_{h}, e-e_{i}\right)+\left(\lambda_{h}, \psi+\delta\left(f+\lambda_{h}\right)-u\right) \\
& +\left(\lambda_{h}, u_{h}-\psi-\delta\left(f+\lambda_{h}\right)\right) \\
= & \left(\nabla u, \nabla\left(e_{i}-e\right)\right)-\left(f, e_{i}-e\right) \\
& +\left(\lambda_{h}, e-e_{i}\right) \\
& +\left(\lambda_{h}, \psi+\delta\left(f+\left[-\Delta u_{h}-f\right]-u\right)\right) \\
& +\left(\lambda_{h}, u_{h}-\psi-\delta\left(f+\lambda_{h}\right)\right) \\
= & \left(\nabla u, \nabla\left(e_{i}-e\right)\right)-\left(f, e_{i}-e\right) \\
& \left.+\left(\lambda_{h}, e-e_{i}\right)+\left(\lambda_{h}, \psi-\delta \Delta u_{h}-u\right)\right) \\
& +\left(\lambda_{h}, u_{h}-\psi-\delta\left(f+\lambda_{h}\right)\right) \\
= & \left(\nabla u, \nabla\left(e_{i}-e\right)\right)-\left(f, e_{i}-e\right) \\
& +\left(\lambda_{h}, e-e_{i}\right)+\underbrace{\left(\lambda_{h}, \psi-u\right)}_{\leq 0} \\
& +\left(\lambda_{h},-\delta\left[\Delta u_{h}+f+\lambda_{h}\right]+u_{h}-\psi\right) .
\end{aligned}
$$

By the same arguments as above, we obtain

Theorem 3 For the stabilized mixed FE-scheme (1819), the a posteriori error estimate

$|e|_{1}^{2} \leq C \sum_{T \in \mathbb{T}_{h}} \varrho_{T}^{2}+N$

holds with $\varrho_{T}$ from (29) and

$N:=\left(\lambda_{h},-\delta\left[\Delta u_{h}+f+\lambda_{h}\right]+u_{h}-\psi\right)$.
We compare the derived a posteriori error estimators with the one presented in [19]. It reads

Theorem 4 For the discretisation error holds the a posteriori error estimate

$|e|_{1}^{2} \leq C\left(\sum_{T \in \mathbb{T}_{h}} \varrho_{T}^{2}+s_{1}+s_{2}\right)$,

where

$s_{1}:=\left\|\left(\psi-u_{h}\right)_{+}\right\|_{1, \Gamma_{C}}^{2}, s_{2}:=\left|\left(\lambda_{H},\left(\psi-u_{h}\right)_{+}\right)_{\Gamma_{C}}\right|$,

and the positive part of a function $g$ is denoted by $g_{+}(x)=$ $\max \{g(x), 0\}$.

\section{Numerical results}

In this section, we discuss numerical examples concerning the stabilization of the mixed problem, efficiency of the solution algorithms, as well as the a posteriori error estimators and adaptive algorithms. In all examples, we take the domain $\Omega=[0,1]^{2}$, a constant force $f=-10$ and $u_{h} \in V_{h}$. The obstacle given by the function $\psi$ is varied throughout the examples.

\subsection{Stabilization}

We begin with the discussion of the stabilization results. For these examples, $\psi=-0.25$ is chosen. In Figure 1 (a), the displacement $u_{h} \in V_{h}$ and in Figure 1 (b) the Lagrange multiplier $\lambda_{H} \in L_{H}=V_{h}$ without stabilization are shown. We observe that the membrane soaks into the obstacle. Furthermore, there are oscillations in the Lagrange multiplier, which has a maximum value that is much too high. We expect it to be at most at the value of 10 . Adding the stabilization term with $\delta=\gamma h^{2}$ and $\gamma=0.03$, we obtain the results presented in Figure 2. There are no more oscillations in the Lagrange parameter, which takes the expected value, and the membrane does not penetrate the obstacle.

Another way to obtain a stable discretization is to use $L_{H} \neq V_{h}$. Here, we choose

$$
L_{H}:=\left\{\mu_{H} \in L^{2}\left(\Gamma_{C}\right) \mid \mu_{H \mid T} \in \mathcal{P}_{0}, \forall T \in \mathbb{T}_{H}\right\} .
$$

The results for $H=h$ are shown in Figure 3. We observe the same behaviour as depicted in Figure 1. Consequently, this discretization is not satisfactory. However, choosing $H=2 h$ leads to a stable discretization, c.f. Figure 4. 


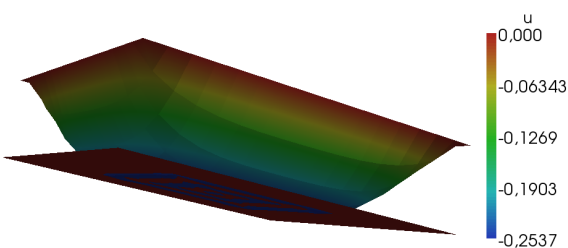

$*_{2}$

(a) Displacement $u_{h}$

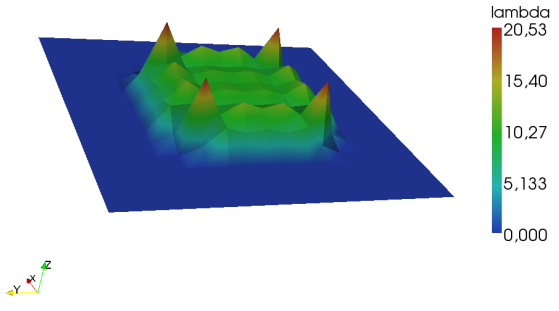

(b) Lagrange multiplier $\lambda_{H}$

Fig. 1 Displacement and Lagrange multiplier for $\lambda_{H} \in V_{h}$ without stabilization

\subsection{Comparison of different solution algorithms}

In Table 1 the number of iterations for different solution techniques are compared, where the obstacle is chosen as $\psi=-0.25$. This choice of the obstacle leads to a large contact zone and thus many nodes are in contact with the obstacle and the number of degrees of freedom of the Lagrange multiplier unequal zero is large, too. In this situation, the Uzawa algorithm and the optimization in the Lagrange multipliers by SQOPT using the Schur complement need a large number of iterations because of the large number of degrees of freedom of the Lagrange multiplier being unequal zero, whereas the cGPSSOR method is very effective due to the fact that many nodes are in contact and are simply projected onto the obstacle. The preconditioned (pc.) Uzawa algorithm is very effective, too. Here, the preconditioned Uzawa is an Uzawa algorithm with an SSOR- and a cg-step as a preconditioner.

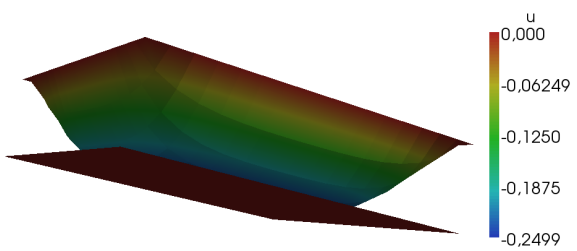

$2 x^{2}$

(a) Displacement $u_{h}$

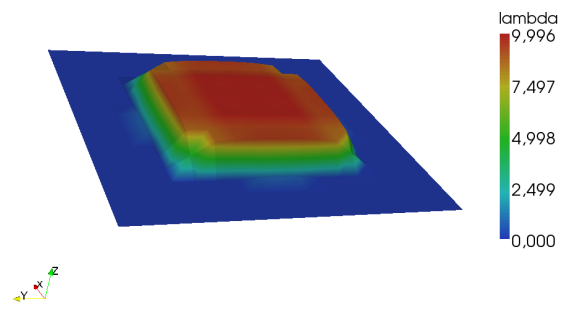

(b) Lagrange multiplier $\lambda_{H}$

Fig. 2 Displacement and Lagrange multiplier for $\lambda_{H} \in V_{h}$ with stabilization

\begin{tabular}{c|c|c|c|c}
\hline Elements & SQOPT & cGPSSOR & Uzawa & pc. Uzawa \\
\hline 64 & 1 & 18 & 12 & 3 \\
256 & 4 & 41 & 75 & 3 \\
1024 & 4 & 138 & 185 & 10 \\
4096 & 12 & 445 & 631 & 15 \\
16384 & 32 & 1530 & 2812 & 50 \\
65536 & 140 & 6046 & 14045 & 45 \\
\hline
\end{tabular}

Table 2 Comparison of the number of iterations of different solution algorithms, $\psi=-0.7$

\begin{tabular}{c|c|c|c|c}
\hline Elements & SQOPT & cGPSSOR & Uzawa & pc. Uzawa \\
\hline 64 & 4 & 11 & 70 & 8 \\
256 & 18 & 20 & 217 & 14 \\
1024 & 86 & 46 & 864 & 30 \\
4096 & 304 & 140 & 3142 & 58 \\
16384 & 1188 & 444 & 16047 & 263 \\
65536 & 4632 & 1534 & 115986 & 428 \\
\hline
\end{tabular}

Table 1 Comparison of the number of iterations of different solution algorithms, $\psi=-0.25$

The contact situation discussed in Table 2 is completely different, since the choice $\psi=-0.7$ yields a small contact zone with few nodes in contact and with 


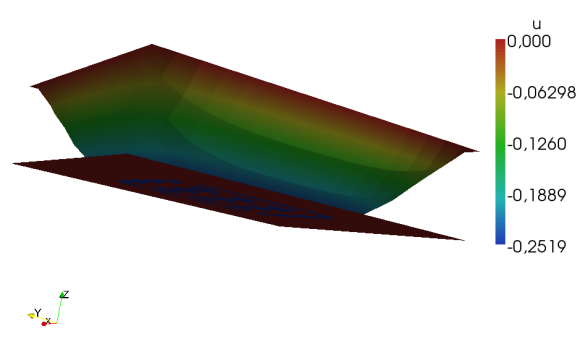

(a) Displacement $u_{h}$

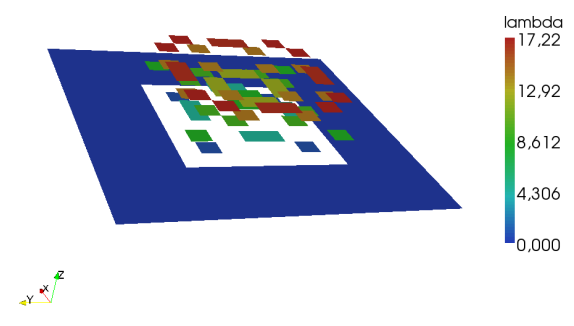

(b) Lagrange multiplier $\lambda_{H}$

Fig. 3 Displacement and Lagrange multiplier for $\lambda_{H} \in L_{H}$ with $H=h$

many degrees of freedom of the Lagrange multiplier being zero. Consequently, the approach using the Schur complement (SQOPT) is very effective and the cGPSSOR method is less effective. The Uzawa is better with less contact but still the one with the most iterations whereas number of the preconditioned Uzawas iterations is smaller than the one of SQOPT. The advantages of the Schur complement approach become even more obvious, when Signorini problems are considered, which include only obstacles on the boundary, c.f. [19].

\subsection{Adaptivity with different error estimators}

This section is devoted to the test and comparison of the three discussed a posteriori error estimates (26), (32) and (34). At first, we choose a smooth obstacle with $\psi=-0.25$. In Figure 5, the adaptive meshes generated on the basis of the three error estimators are depicted. The adaptive meshes created by the estimators (32) and (34), which take the Lagrange multiplier into account, are similar. The small differences occur on the one hand since the adaptive refinement strategies vary and on the other hand because of the stabilization of the Lagrange multiplier in Figure 5 (c) by

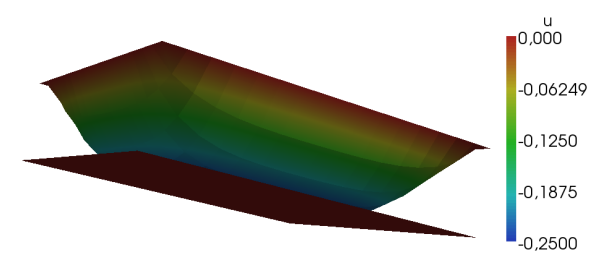

${ }^{2}{ }^{2}{ }^{2}$

(a) Displacement $u_{h}$

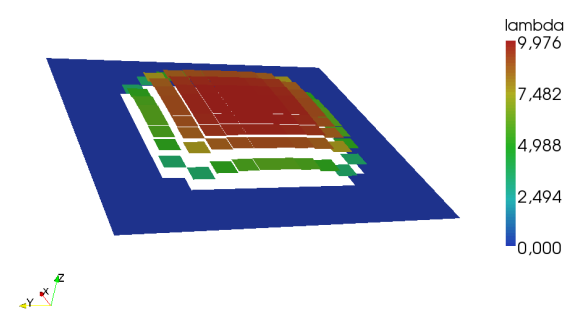

(b) Lagrange multiplier $\lambda_{H}$

Fig. 4 Displacement and Lagrange multiplier for $\lambda_{H} \in L_{H}$ with $H=h 2$

coarsening the mesh for it, which demands a so called patch structure of the mesh. We observe large deviations between Figure 5 (a) as well as Figure 5 (b) and (c). The a posteriori error estimator (26) leads to a well refined contact zone, whereas the estimators (32) and (34) yield to coarse grids in the interior of the contact zone. That is more efficient because in the contact zone there does not happen anything that causes observable errors. Consequently, we need less unknowns with the improved estimator. The reason for this behaviour is that the residual $\left\|\Delta u_{h}+f\right\|_{T}$ of the error estimator (26) is large in the contact zone due to the variational inequality. Whereas the residual $\left\|\Delta u_{h}+f+\lambda_{H}\right\|_{T}$ of the error estimators (32) and (34) is small.

In Figure 6, the estimated error of the a posteriori error estimators (26) and (32) is compared. While having the same convergence rate, the error estimated by (32) is smaller than the one estimated by (26). Since both estimators provide upper bounds of the discretization error, the estimation of (32) is more accurate.

In Figure 7 the estimated convergence rates for uniform refinement and for adaptive refinement based on the a posteriori error estimates (32) and (34) are depicted. Since we consider a smooth obstacle here, the 


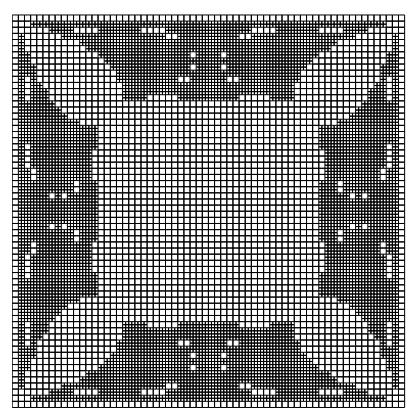

(a) Estimator (26)

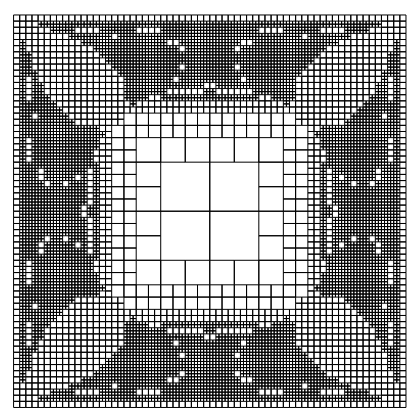

(b) Estimator (32)

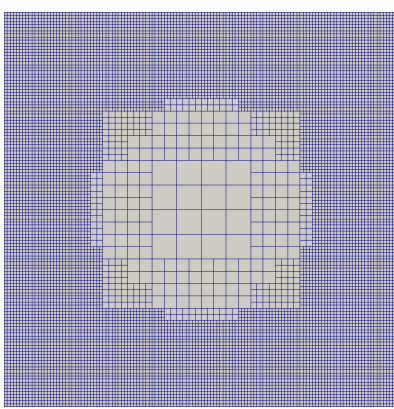

(c) Estimator (34)

Fig. 5 Adaptive meshes generated on the basis of different error estimators

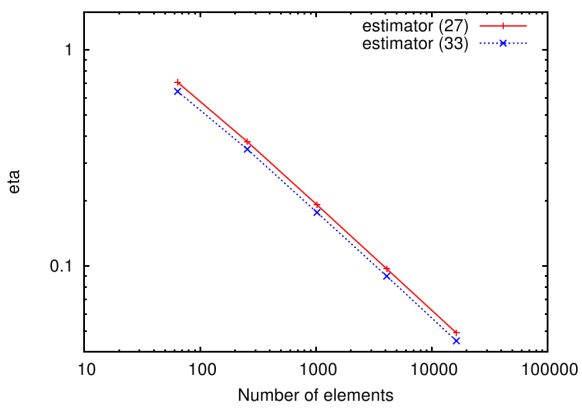

Fig. 6 Comparison of the estimated error of the estimators (26) and (32)

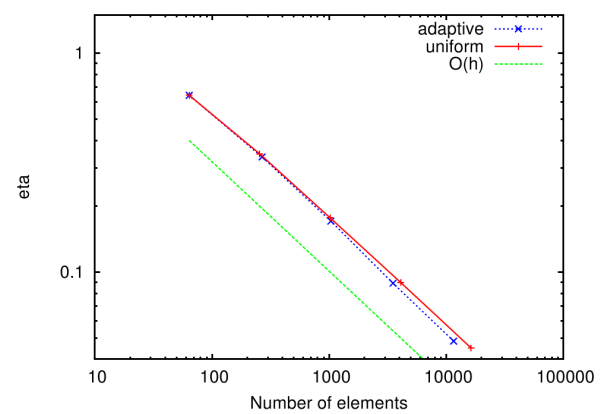

(a) Estimator (32)

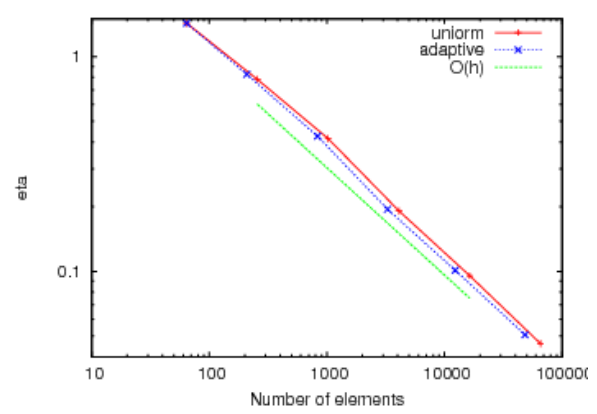

(b) Estimator (34)

Fig. 7 Comparison of adaptive and global uniform refinement for a smooth obstacle

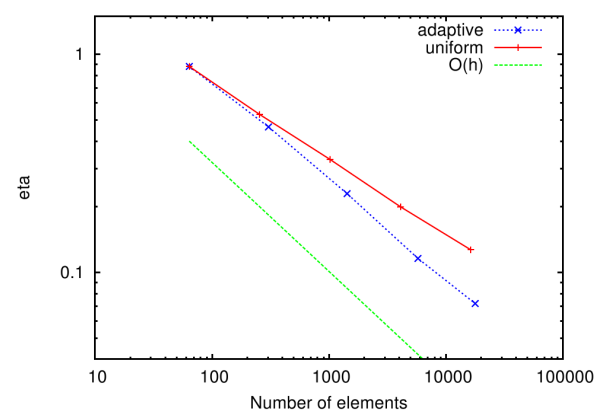

(a) Estimator (32)

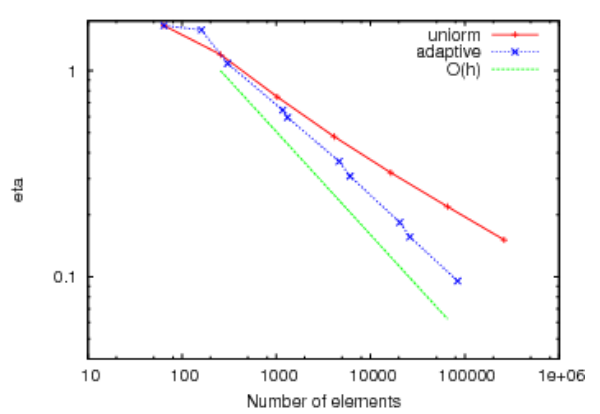

(b) Estimator (34)

Fig. 8 Comparison of adaptive and global uniform refinement for a discontinuous obstacle

uniform approach leads to the optimal convergence rate of $\mathcal{O}(h)$. The adaptive refinement converges also of optimal order $\mathcal{O}(h)$, but the same error is achieved with less unknowns.

The advantages of adaptive refinement become more obvious, if a discontinuous obstacle is considered. We choose $\psi=-0.25$ if $x, y \in[0.5-0.125,0.5+0.125]$ and $\psi=-1$ elsewhere. In Figure 8, the convergence results based on the a posteriori error estimates (32) and (34) are shown. The adaptive refinement clearly leads to the optimal convergence rate of $\mathcal{O}(h)$. However, the convergence rate of the uniform refinement is reduced. 


\section{Conclusions and Outlook}

In this paper, we have presented and compared different mixed and adaptive finite element discretizations for the obstacle problem. As usual in mixed finite element methods, ensuring the stability is crucial. Two different methods to this end have been discussed: The first approach is to modify the trial space for the Lagrange multiplier, which can be difficult to realize but allows for the direct use of the a posteriori error estimates. Adding a consistent stabilization term in the mixed problem is the second method. This approach permits the use of the same finite element approach for the primal and the dual variable. However, the additional term has to be considered in the a posteriori error estimates. In the derivation of the a posteriori error estimates, consistency is the central point to achieve accurate error estimates, which lead to effective adaptive discretizations. The comparison of different solution techniques for the discrete problem shows that the efficiency of the algorithms strongly depends on the number of degrees of freedom in contact with the obstacle.

The obstacle problem is a model problem for three dimensional Signorini contact problems. It allows the study of effects, which are of great importance for the discretization of Signorini problems. Consequently, the transfer of the presented results to Signorini problems is the next step. Moreover, frictional effects play an important role in many engineering problems and have to be included.

Acknowledgements This research work was supported by the Deutsche Forschungsgemeinschaft (DFG) within the Priority Program 1480, Modelling, Simulation and Compensation of Thermal Effects for Complex Machining Processes, with the grant numbers BI 498/24-1, BL 256/11-1, and SU $245 / 6-1$.

\section{References}

1. Ainsworth, M., Oden, J.T., Lee, C.Y.: Local a posteriori error estimators for variational inequalities. Numer. Meth. Partial Differ. Equ. 9, 23-33 (1993)

2. Bartels, S., Carstensen, C.: Averaging techniques yield reliable a posteriori finite element error control for obstacle problems. Numer. Math. 99, 225-249 (2004)

3. Blum H., Braess, D., Suttmeier, F.T.: A cascadic multigrid algorithm for variational inequalities. Comput. Visual. Sci. 7, 153-157 (2004)

4. Braess, D.: A posteriori error estimators for obstacle problems - another look. Numer. Math. 101, 415-421 (2005)

5. Braess, D., Carstensen, C., Hoppe, R.H.W.: Convergence analysis of a conforming adaptive finite element method for an obstacle problem. Numer. Math. 107, 455-471 (2007)

6. Braess, D., Carstensen, C., Hoppe, R.H.W.: Error reduction in adaptive finite element approximations of elliptic obstacle proplems. J. Comput. Math. 27(2-3), 148-169 (2009)
7. Brézis, H.: Problèmes unilatéraux. J. Math. Pur. Appl. 51, 1-168 (1972)

8. Brezzi, F., Hager, W., Raviart, P.A.: Error estimates for the finite element solution of variational inequalities. Numer. Math. 28, 431-443 (1977)

9. Chen, Z., Nochetto, R.: Residual type a posteriori error estimates for elliptic obstacle problems. Numer. Math. 84, 527-548 (2000)

10. Falk, R.S.: Error estimates for the approximation of a class of variational inequalities. Math. Comp. 28, 963-971 (1974)

11. Glowinski, R.: Numerical methods for nonlinear variational problems. Springer-Verlag (1983)

12. Hoppe, R.H.W., Kornhuber, R.: Adaptive multilevel methods for obstacle problems. SIAM J. Numer. Anal. 31, 301-323 (1994)

13. Hughes, T.J.R., Franca, L.P., Balestra, M.: A new finite element formulation for computational fluid dynamics: V. Circumventing the Babuška-Brezzi condition: A stable Petrov-Galerkin formulation for the Stokes problem accommodating equal order interpolation. Comp. Meth. Mech. Eng. 59, 85-99 (1986)

14. Franca, L.P., Stenberg, R.: Error analysis of some Galerkin least squares methods for the elasticity equations. SIAM J. Numer. Anal. 28 (1991)

15. Johnson, C.: Adaptive finite element methods for the obstacle problem. Math. Models Meth. Appl. Sci. 2, 483-487 (1992)

16. Laursen, T. A.: Computational contact and impact mechanics. Springer, Berlin (2002)

17. Lions, J.L., Stampacchia, G.: Variational inequalities. Comm. Pure Appl. Math. 20, 493-519 (1967)

18. Nochetto, R.H., Siebert, K., Veeser, A.: Pointwise a posteriori error control for elliptic obstacle problems. Numer. Math. 95, 163-195 (2003)

19. Schröder, A.: Fehlerkontrollierte adaptive $h$ - und $h p$ Finite-Elemente-Methoden für Kontaktprobleme mit Anwendungen in der Fertigungstechnik. Dissertation Universität Dortmund (2005)

20. Schröder, A., Blum, H., Rademacher, A., Kleemann, H.: Mixed FEM of higher order for contact problems with friction. IJNAM, Int. J. Numer. Anal. Modeling, 8(2), 302-323 (2011)

21. Siebert, K., Veeser, A.: A unilaterally constrained quadratic minimization with adaptive finite elements. SIAM J. Optim. 18, 260-289 (2007)

22. Suttmeier, F.T.: Numerical solution of variational inequalities by adaptive finite elements. Vieweg+Teubner Verlag, Wiesbaden (2008)

23. Veeser, A.: Efficient and reliable a posteriori error estimators for elliptic obstacle problems. SIAM J. Numer. Anal. 39, 146-167 (2001)

24. Wohlmuth, B.: Variationally consistent discretization schemes and numerical algorithms for contact problems. Acta Numerica 20, 569-734 (2011)

25. Wriggers, P.: Computational contact mechanics. John Wiley \& Sons Ltd, Chichester (2002) 